\title{
Online communities for breast cancer survivors: a review and analysis of their characteristics and levels of use
}

\author{
Jacqueline L. Bender • M. Carolina Jimenez-Marroquin • \\ Lorraine E. Ferris • Joel Katz • Alejandro R. Jadad
}

\begin{abstract}
Purpose Online communities have been heralded as one of the most promising health resources on the Internet. The purpose of this study was to identify the characteristics and levels of use of online communities for breast cancer survivors.

Methods Using Google, we identified websites with a string of computer-mediated communication terms and individual queries of three to five words of online community terms. This was complemented by a review of website resource lists and personal libraries. Two reviewers independently extracted information on their general characteristics and
\end{abstract}

J. L. Bender $\cdot$ L. E. Ferris $\cdot$ A. R. Jadad

Dalla Lana School of Public Health, University of Toronto,

Toronto, Ontario, Canada

J. L. Bender $(\bowtie) \cdot$ M. C. Jimenez-Marroquin • A. R. Jadad Centre for Global eHealth Innovation,

University Health Network,

190 Elizabeth Street,

Toronto M5G 2C4 Ontario, Canada

e-mail: jbender@ehealthinnovation.org

J. L. Bender · A. R. Jadad

ELLICSR Health Wellness and Cancer Survivorship Centre, University Health Network,

Toronto, Ontario, Canada

A. R. Jadad

Department of Health Policy, Management and Evaluation,

University of Toronto,

Toronto, Ontario, Canada

J. Katz

Department of Psychology, York University,

Toronto, Ontario, Canada number of members and message board posts. A coding scheme guided content analysis.

Results We found 111 websites. Most sites ( $n=64,65.8 \%)$ had a broad focus (e.g., health, cancer, or general). One third $(n=38,34.2 \%)$ were exclusive to breast cancer and 11 catered to specific disease characteristics. The majority were American $(n=79,75.2 \%)$, nonprofit $(55.0 \%)$, and moderated $(69.5 \%)$. Most moderators $(85.7 \%)$ were staff or community members; eight sites were moderated by health professionals. Greater than one-third of sites $(n=40,36 \%)$ were initiated by breast cancer survivors or loved ones. Breast cancer-specific sites contained a total of 4,186,275 posts. One-third $(n=10)$ contained $93.4 \%$ of posts, displaying over 100,000 posts each. As of April 3, 2012, eight sites were discontinued.

Conclusions There is a wide range of online communities available for breast cancer survivors with extensive archives of personal illness experiences. Future efforts should focus on identifying the factors that determine their success and effectiveness.

Keywords Online communities · Breast cancer · Health information $\cdot$ Internet $\cdot$ Social support

\section{Background}

The Internet has changed the way people seek and engage with health information. Although health professionals, family, and friends remain the preferred sources of health information, online resources, including patient experiences are a significant source of health information and support [1, 2]. Surveys conducted in 2010 indicate that at least $80 \%$ of North Americans use the Internet regularly, that six in ten 
have looked online for health information $[1,3]$ and that one in four Americans have read about someone else's health experience in an online community [1].

Online communities have been defined as "virtual social space(s) where people come together to get and give information or support, to learn, or to find company" [4]. The terms "online," "virtual," "Internet-mediated-," "web-based-," "group," "community," or "network" are used interchangeably in the literature to refer to the same concept. Initially, online communities were supported by mailing list and message board software. More recently, they have formed around and across blogs, wikis, and social network sites Web 2.0 software applications, which allow the creation and exchange of content by anyone in an open collaborative fashion [5].

Breast cancer survivors have been identified as one of the groups most likely to use online communities as a health resource [6]. Although there is limited empirical evidence concerning their effects [7], numerous descriptive studies indicate that online communities have empowering benefits. Such groups have been shown to provide breast cancer survivor reassurance, a sense of community and hope for the future, reduce feelings of stress and uncertainty, validate concerns ignored by health-care professionals, enable breast cancer survivors to become better informed and able to manage their condition, and prepare breast cancer survivors for their interactions with the health-care professionals [8-13].

However, we know little about the amount and characteristics of online communities for people living with breast cancer, including which ones are most popular and why. A review conducted in 2003 identified a total of 546 online cancer communities but did not characterize them by cancer type or report their levels of activity [14]. Two studies that attempted to evaluate the quality of information in a sample of breast cancer websites $[15,16]$ documented the type of communication software that the sites provided but also did not report on their levels of use. One study [16] concluded that the most popular breast cancer websites (as determined by Google rank) were correlated with type rather than quality of content and contained message board software. All of these studies were published in the early 2000 s, well before the emergence of Web 2.0 and the subsequent social media revolution.

We need to gain a better understanding on the most popular online communities so that we can effectively target our effectiveness research and guide patients to useful resources. One way to assess the popularity of an online community is to determine its activity, as measured by the number of user-generated posts [17]. Therefore, the purpose of this study was to identify websites that provide English language online communities for breast cancer survivors and to synthesize their general characteristics and patterns of use.

\section{Methods}

Data sources

Websites were identified through a search of Google (www.google.ca) accessed on December 23, 2009, because it represented the leading search engine used by $85 \%$ of the global market share at that time [18].

Inclusion criteria

Websites were included if they

- Contained communication technology (e.g., mailing list, message board, chat room, blog, wiki) that served as a forum for breast cancer related topics;

- Contained content (including that contributed by users) in English;

- Were active, as defined as having more than one post contributed by members from the date the search was conducted (December 23, 2009) to the date the total number of the members and posts was documented (October 26, 2010); and

- Were publicly available.

Excluded websites

- Single author blogs (as opposed to sites that provided facilities for any visitor to create and sustain a personal blog);

- Websites that contained wikis as the only form of computer-mediated communication if they were not being used for breast cancer-themed discussion; and

- Website aggregators (i.e., websites that pool and publish other websites).

\section{Search strategy}

We used two complementary search strategies to locate relevant websites (Appendix 2). The first approach, modeled after a typical academic literature search, used Boolean operators (e.g., "AND" and "OR") to connect a string of computer-mediated communication technology terms; all accessible hits were investigated. The second approach, modeled after a typical Google query, which consists of an average of four words [19], included nine separate searches of different types of online communities (e.g., breast cancer message boards). The first 100 hits from each of the nine searches were investigated after it was concluded from the first approach that there would likely be about 100 relevant sites in total. Both search strategies were applied to "anywhere on a page." Two of us (JLB and MCJM) independently assessed the eligibility of all search results. One of us (JLB) reviewed the resource lists of retrieved websites, as 
well as personal libraries for other potentially eligible websites to include in the review. Disagreements were resolved by consensus in all cases.

Data collection and analysis

Two of us (JLB and MCJM) independently extracted information on the following:

- General characteristics (e.g., URL, launch date, purpose, country of origin, initiator, affiliation, and moderation)

- Number and type of communication technologies (e.g., mailing list, asynchronous message board, chat room, blog, wiki)

- Social networking features (e.g., ability to construct a public profile and view the profiles and networks of other members)

- Total number of registered users and posts as of October 26, 2010, (11 months after the sites were identified and their general characteristics were extracted for analysis)

- Status as of April 3, 2012

We developed a coding scheme to classify the purpose (breast cancer exclusively, cancer, any health issue, or any general topic), affiliation (e.g., for-profit organization, nonprofit organization, university or health care organization, or unaffiliated individual or group), and initiator (former cancer survivor/ family caregiver, health professional, health research, charity, or unknown). Sites were classified as moderated if they claimed to be so or contained evidence of moderation from site administrators or suggestive activities in the message board (e.g., posts that welcomed new members, individuals who responded to member requests or questions, or who stimulated discussion).

We contacted site administrators, where possible, to request the total number of registered members, posts, and date of launch, if it was not published on the site. We obtained permission from site owners to publish any publicly unavailable information. The total number of registered members and message board posts is summarized from breast cancer-specific sites only because this information was not consistently available from multipurpose sites. Only $21.9 \%$ of multipurpose sites disclosed the total number of posts, and only $2.7 \%$ disclosed their membership numbers.

We met to review all data extracted and coded. Disagreements were resolved through consensus, including crosschecking the original source and involving a third reviewer when necessary.

Evidence tables were produced to summarize the information extracted from the websites and obtained from the site administrators. We calculated descriptive statistics using the software package SPSS version 17 (IBM Corporation, Somers, NY, USA).

This study was a component of a larger research study for which ethical approval was obtained from our Institution's Research Ethics Board.

\section{Results}

The total yield from the two Internet search strategies was 1,714. As shown in Fig. 1, 153 websites were subjected to preliminary analysis and 111 were included in the final analysis. The complete inventory of online communities used in this study is presented in Table 1.

Characteristics of included websites

\section{Purpose}

Characteristics of the websites are shown in Table 2. The majority $(65.8 \%)$ of sites were multipurpose. These included general health sites (www.mdjunction.com), cancer sites

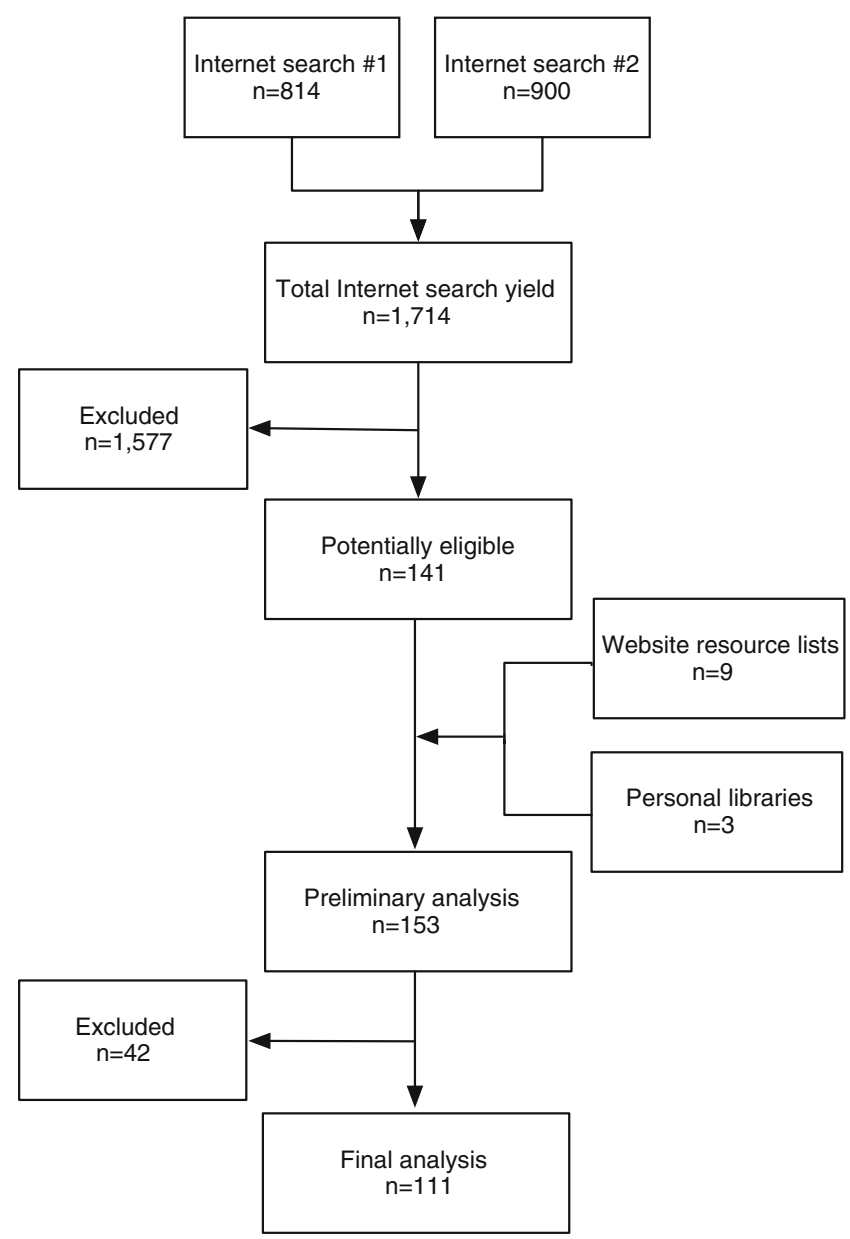

Fig. 1 Flow diagram of included websites 
Table 1 Inventory of online communities for breast cancer survivors

\begin{tabular}{|c|c|c|c|}
\hline & Name & URL & Focus \\
\hline 1 & Association for Online Cancer Resources (ACOR) & www.acor.org & Cancer \\
\hline \multirow[t]{2}{*}{2} & Action Breast Cancer Ireland & $\begin{array}{l}\text { www.cancer.ie/action/ } \\
\text { http://www.cancer.ie/cancer-information/breast-cancer }\end{array}$ & Breast cancer \\
\hline & & http://forum.cancer.ie/phpBB3/ & \\
\hline 3 & All About You & www.allaboutyou.com & General purpose \\
\hline 4 & AOL & http://messageboards.aol.com & General purpose \\
\hline 5 & Aussie Breast Cancer Forum & www.bcaus.org.au/ & Breast cancer \\
\hline $6^{\mathrm{a}}$ & Avon Crusade & Closed & Breast cancer \\
\hline 7 & B-mail & www.bci.org.au & Breast caner \\
\hline 8 & BC Advisor & www.bcadvisor.com/ & Breast cancer \\
\hline 9 & BC Mets & www.bcmets.org & Breast cancer (metastatic) \\
\hline 10 & BC Pals & www.bcpals.org.uk & Breast cancer \\
\hline 11 & BC Support & www.bcsupport.org & Breast cancer \\
\hline 12 & Bebo & www.bebo.com & General purpose \\
\hline 13 & Bella Online Forums & www.bellaonline.com & General purpose \\
\hline 14 & Be Bright Pink & www.bebrightpink.org & $\begin{array}{l}\text { Breast cancer (high } \\
\text { risk young women) }\end{array}$ \\
\hline 15 & Bizymoms & www.bizymoms.com & General purpose \\
\hline 16 & Blog for a cure & www.blogforacure.com & Cancer \\
\hline 17 & Bosom Buddies & http://bosombuddies.cfsites.org/ & Breast cancer \\
\hline 18 & Breast Buddies & www.breastbuddies.co.za/forum & Breast cancer \\
\hline 19 & Breastcancer.org & www.breastcancer.org & Breast cancer \\
\hline 20 & Breast Cancer Awareness & www.breastcancerawareness.com & Breast cancer \\
\hline 21 & Breast Cancer Care & www.breastcancercare.org.uk & Breast cancer \\
\hline 22 & Breast Cancer Mailing List & www.bclist.org & Breast cancer \\
\hline $23^{\mathrm{b}}$ & $\begin{array}{l}\text { Breast Cancer Network of Strength (Rebranded: } \\
\text { Y-Me Breast Cancer Support Today) }\end{array}$ & http://www.y-me.org/ & Breast cancer \\
\hline 24 & Breast Cancer Now What & www.breastcancernowwhat.ca & $\begin{array}{l}\text { Breast cancer } \\
\text { (young women) }\end{array}$ \\
\hline 25 & Breast Cancer Support New Zealand & www.breast.co.nz/ & Breast cancer \\
\hline 26 & Breast Cancer Support UK & www.breastcancersupport.co.uk & Breast cancer \\
\hline $27^{\mathrm{a}}$ & Breast Friends & Closed & Breast cancer \\
\hline 28 & Breast Health Online & www.breasthealthonline.org & Breast cancer \\
\hline 29 & Breast Reconstruction & www.breastreconstruction.org & $\begin{array}{l}\text { Health (breast } \\
\text { reconstruction) }\end{array}$ \\
\hline 30 & Cancer Buddies Network & www.cancerbuddiesnetwork.org & Cancer \\
\hline 31 & Cancer Survivors Network & http://csn.cancer.org/forum & Cancer \\
\hline 32 & Cancer Care & www.cancercare.org & Cancer \\
\hline 33 & Cancer Chat UK & www.cancerchat.org.uk & Cancer \\
\hline 34 & Cancer Compass & www.cancercompass.com & Health \\
\hline 35 & Cancer Connections & www.cancerconnections.com.au & Cancer \\
\hline 36 & Cancer Focus & www.cancerfocus.org & Cancer \\
\hline 37 & Cancer Forums & www.cancerforums.net & Cancer \\
\hline 38 & Cancer Match & www.cancermatch.com/ & Cancer \\
\hline 39 & Cancer Support & http://cancersupport.aarogya.com & Cancer \\
\hline $40^{\mathrm{a}}$ & Cancer Treatment Forums & Closed & Cancer \\
\hline 41 & Care Pages & www.carepages.com & Health \\
\hline 42 & Caring 4 Cancer & www.caring 4 cancer.com & Cancer \\
\hline 43 & Caring Voices & www.caringvoices.ca & Cancer \\
\hline 44 & Cure Today & www.curetoday.com & Cancer \\
\hline
\end{tabular}


Table 1 (continued)

\begin{tabular}{|c|c|c|c|}
\hline & Name & URL & Focus \\
\hline 45 & Cure Zone & www.curezone.com & Cancer \\
\hline 46 & Daily Strength & www.dailystrength.org & Health \\
\hline 47 & DIEP Breast Reconstruction & www.diepbreastreconstruction.org/forum/ & $\begin{array}{l}\text { Breast cancer } \\
\text { (post-mastectomy } \\
\text { reconstruction) }\end{array}$ \\
\hline 48 & Discovery Health & http://health.discovery.com/conversations/conversations.html & Health \\
\hline 49 & eHealth Forums & http://ehealthforums.com & Health \\
\hline 50 & EmpowHER & www.empowher.com & Health \\
\hline 51 & Everyday Health & www.everydayhealth.com & Health \\
\hline 52 & Experience Project & www.experienceproject.com & Health \\
\hline 53 & Facebook & www.facebook.com & General \\
\hline 54 & Facing Our Risk of Cancer Empowered (FORCE) & www.facingourrisk.org & $\begin{array}{l}\text { Breast and Ovarian } \\
\text { cancer (hereditary) }\end{array}$ \\
\hline 55 & Friends in Need & www.friendsinneed.com & Breast cancer \\
\hline 56 & Friends in Touch & www.friendsintouch.net & Breast cancer \\
\hline 57 & Healia Communities & http://communities.healia.com & Health \\
\hline 58 & Healing well & www.healingwell.com & Health \\
\hline 59 & Health & www.health.com & Health \\
\hline 60 & Health Boards & www.healthboards.com & Health \\
\hline 61 & Health Central & www.healthcentral.com & Health \\
\hline 62 & Health Forums & www.health-forums.com & Health \\
\hline 63 & Health Front & www.healthfront.com & Health \\
\hline 64 & Health Talk & www.healthtalkonline.org & Health \\
\hline 65 & Her 2 Support & www.her2support.org & $\begin{array}{l}\text { Breast cancer } \\
\text { (Her } 2 \text { positive) }\end{array}$ \\
\hline 66 & Hystersisters & www.hystersisters.com & Health (hysterectomy) \\
\hline 67 & Inflammatory Breast Cancer Support & www.ibcsupport.org & Breast cancer \\
\hline 68 & Imaginis & www.imaginis.com & Health \\
\hline $69^{\mathrm{a}}$ & iMedix & Closed & Health \\
\hline 70 & Inspire & www.inspire.com & Health \\
\hline 71 & iVillage & www.ivillage.com & General \\
\hline 72 & Living Beyond Breast Cancer & www.lbbc.org & Breast cancer \\
\hline 73 & Lymphedema People & www.lymphedemapeople.com & Health (lymphedema) \\
\hline 74 & MD Anderson & www.mdanderson.org/ & Cancer \\
\hline 75 & MD Junction & www.mdjunction.com & Health \\
\hline 76 & MSN Health \& Fitness & http://health.msn.com & Health \\
\hline 77 & MacMillan & www.macmillan.org.uk & Cancer \\
\hline 78 & Make Me Heal & www.makemeheal.com & Health (plastic surgery) \\
\hline 79 & Med Help & www.medhelp.org & Health \\
\hline 80 & Medpedia & www.medpedia.com & Health \\
\hline $81^{\mathrm{a}}$ & Moms Like Me & Closed & General \\
\hline 82 & My Lifetime & www.mylifetime.com & General \\
\hline 83 & MySpace & www.myspace.com & General \\
\hline 84 & National Breast Cancer Foundation & www.nationalbreastcancer.org & Breast Cancer \\
\hline 85 & Net Doctor & www.netdoctor.co.uk & Health \\
\hline 86 & No Surrender & www.nosurrenderbreastcancersurvivorforum.org & Breast cancer \\
\hline 87 & Oncochat & www.oncochat.org & Cancer \\
\hline $88^{\mathrm{a}}$ & $\begin{array}{l}\text { Oncology Channel - (Rebranded } \\
\text { Health Communities) }\end{array}$ & www.healthcommunities.com (Removed online community) & Cancer/Health \\
\hline 89 & Pink Link & www.pink-link.org/ & Breast cancer \\
\hline
\end{tabular}


Table 1 (continued)

\begin{tabular}{|c|c|c|c|}
\hline & Name & URL & Focus \\
\hline 90 & Pink Ribbon & www.pinkribbon.org & Breast cancer \\
\hline 91 & Pink Ribbon Girls & www.pinkribbongirls.org & Breast cancer \\
\hline 92 & Revolution Health & www.revolutionhealth.com & Health \\
\hline $93^{\mathrm{a}}$ & Sharing Strength & www.sharingstrength.ca (archived) & Breast cancer \\
\hline 94 & Susan G Komen Breast Cancer Foundation & http://apps.komen.org/Forums/default.aspx & Breast cancer \\
\hline 95 & The Breast Cancer Site & http://www.thebreastcaresite.com/tbcs/ & Breast cancer \\
\hline 96 & Topix & www.topix.com & General \\
\hline 97 & Triple Negative Breast Cancer Foundation & www.tnbcfoundation.org & $\begin{array}{l}\text { Breast cancer } \\
\text { (triple negative) }\end{array}$ \\
\hline 98 & Twitter & www.twitter.com & General \\
\hline 99 & Web MD & www.webmd.com & Health \\
\hline 100 & Wego Health & www.wegohealth.com & Health \\
\hline $101^{\mathrm{b}}$ & $\begin{array}{l}\text { Wellness Community (Rebranded: } \\
\text { Cancer Support Community) }\end{array}$ & www.cancersupportcommunity.org/ & Cancer \\
\hline 102 & Well Sphere & www.wellsphere.com & Health \\
\hline 103 & Wiki Cancer & www.wikicancer.org/ & Cancer \\
\hline $104^{\mathrm{b}}$ & $\begin{array}{l}\text { Willow-Talk (Rebranded: Willow } \\
\text { Breast Cancer Support) }\end{array}$ & www.willow.org & Breast cancer \\
\hline 105 & Women's Health & www.womens-health.com & Health \\
\hline 106 & Women's Health Matters & $\begin{array}{l}\text { http://www.womenshealthmatters.ca/ubbthreads7/ } \\
\text { ubbthreads.php }\end{array}$ & Health \\
\hline $107^{\mathrm{a}}$ & Wrong Diagnosis & Closed & Health \\
\hline 108 & Yahoo & http://health.dir.groups.yahoo.com & General \\
\hline 109 & Young Survival Coalition & www.youngsurvival.org/ & $\begin{array}{l}\text { Breast cancer } \\
\text { (young women) }\end{array}$ \\
\hline 110 & YouTube & www.youtube.com & General \\
\hline 111 & Yuku & www.yuku.com & General \\
\hline
\end{tabular}

${ }^{\text {a }}$ Site has closed down or discontinued its online community

${ }^{\mathrm{b}}$ Site has been rebranded

(www.acor.org), or general purpose sites (www.facebook. com). About one-third of sites (34.2\%) were exclusively for people with breast cancer and, of these, 11 catered to certain disease characteristics (e.g., metastatic) or demographics of breast cancer survivors (e.g., young).

\section{Affiliation and initiator}

Forty-five percent of the online communities were for-profit commercial sites (e.g., affiliated with a pharmaceutical company or product manufacturer). Only seven sites were affiliated with a university or health care institution and, of these, two were affiliated with a cancer treatment center, namely Princess Margaret Hospital in Toronto, Canada, and MD Anderson Cancer Centre in Houston, USA. Fifty-eight percent of sites included information on the individual or group that spearheaded its creation. The majority of these sites $(69.0 \%)$ were initiated by, or on behalf of, a breast cancer survivor or an informal caregiver (e.g., spouse, partner, child, or friend).

\section{Launch dates}

Forty-three of the multipurpose sites $(58.9 \%)$ provided their launch date. Of these, roughly $37 \%$ were launched between 1994 and 1999, $25.5 \%$ between 2000 and 2004, and the remaining $37 \%$ between 2005 and 2009 (data not shown).

Twenty-two of the breast cancer-specific sites $(57.9 \%)$ provided their launch date. The majority of these sites (60.6 \%) were launched between 2004 and 2008 (Fig. 2).

\section{Communication technology}

The majority of websites contained message boards as their main communication tool $(91.9 \%)$ and provided their users with the ability to construct a public or semipublic profile $(82.7 \%)$. Nearly half $(44.8 \%)$ allowed their users to make visible their social networks and thus could be classified as a social network sites. 
Table 2 Characteristics of included websites

\begin{tabular}{|c|c|}
\hline Characteristics & Number $(\%)$ \\
\hline \multicolumn{2}{|l|}{ Purpose $(n=111)$} \\
\hline Breast cancer exclusively & $38(34.2)$ \\
\hline Breast cancer forum on a health site & $36(32.4)$ \\
\hline Breast cancer forum on a cancer site & $21(18.9)$ \\
\hline Breast cancer forum on a general site & $16(14.4)$ \\
\hline \multicolumn{2}{|l|}{ Niche breast cancer communities $(n=11)$} \\
\hline Young women & 4 \\
\hline Breast reconstruction & 2 \\
\hline Hereditary breast cancer & 1 \\
\hline Her2 positive breast cancer & 1 \\
\hline Inflammatory breast cancer & 1 \\
\hline Metastatic breast cancer & 1 \\
\hline Triple negative breast cancer & 1 \\
\hline \multicolumn{2}{|l|}{ Country of origin $(n=105)$} \\
\hline USA & $79(75.2)$ \\
\hline UK & $9(7.6)$ \\
\hline Canada & $8(7.6)$ \\
\hline Australia & $4(3.6)$ \\
\hline South Africa & $2(1.9)$ \\
\hline India & $1(0.9)$ \\
\hline Ireland & $1(0.9)$ \\
\hline New Zealand & $1(0.9)$ \\
\hline \multicolumn{2}{|l|}{ Affiliation $(n=108)$} \\
\hline Commercial & $50(45.0)$ \\
\hline Nonprofit organization & $32(29.6)$ \\
\hline Individual or Group & $19(17.6)$ \\
\hline University or health care institution & $7(6.5)$ \\
\hline \multicolumn{2}{|l|}{ Initiator $(n=58)$} \\
\hline Former patient or caregiver & $40(69.0)$ \\
\hline Health care professional & $10(17.2)$ \\
\hline National Charity & $6(10.3)$ \\
\hline Patient and health care professional & $1(1.7)$ \\
\hline Health researcher & $1(1.7)$ \\
\hline \multicolumn{2}{|l|}{ Communication Features $(n=111)$} \\
\hline Message boards (asynchronous) & $102(91.9)$ \\
\hline Blogs (multiuser) & $42(37.8)$ \\
\hline Chat rooms (synchronous) & $21(18.9)$ \\
\hline Mailing lists & $5(4.5)$ \\
\hline Wikis & $3(2.7)$ \\
\hline \multicolumn{2}{|l|}{ Social networking features } \\
\hline Profiles $(n=110)$ & $91(82.7)$ \\
\hline Create and view friend lists $(n=105)$ & $47(44.8)$ \\
\hline \multicolumn{2}{|l|}{ Moderation $(n=111)$} \\
\hline Yes & $77(69.4)$ \\
\hline \multicolumn{2}{|l|}{ Moderator $(n=77)$} \\
\hline Website staff or volunteers exclusively & $66(85.7)$ \\
\hline Website staff and health care professionals & $8(10.4)$ \\
\hline Health Care professionals exclusively & $3(3.9)$ \\
\hline
\end{tabular}

\section{Moderation}

The majority of websites (69.4\%) were moderated. Most sites $(85.7 \%)$ were moderated by site staff alone or in combination with community members. Only eight sites were moderated by health-care professionals alone, or in combination with website staff. Fifteen sites contained additional features that were moderated by health professionals such as an "Ask the Expert" message board service. Three sites employed a "notice and take down" policy, also known as "reactive moderation" relying on the site members to alert the site administrators of inappropriate use. Only one website (Cancer Chat UK) stated that they screened the message board post accuracy before posting them.

\section{Level of activity of breast cancer-specific sites}

Information on membership and total number of posts was available for 57.9 and $78.9 \%$ of breast cancer-specific sites, respectively. As shown in Table 3, these sites reported a total of 140,162 registered members and 4,186,275 posts. One third of breast cancer-specific sites contained the majority of posts (Fig. 3). The top ten most active breast cancer-specific sites (Table 4) each reported more than 100,000 posts. Four of them catered to a specific type or experience of breast cancer.

\section{Status}

As of April 3, 2012 eight online communities were discontinued - six had shut down, one removed its online community, and another disabled its community functions but archived its content. We successfully contacted two of these sites to inquire about their closure. One site reported that their online community was discontinued because of inactivity and spam. The other site cited changes in the leadership and strategic focus of the funding organization, along with funding pressures and a perceived need to keep pace with the rapidly changing social media landscape.

\section{Discussion}

This study has generated the first known systematic review and characterization of online resources for cancer survivors. In doing so, it has revealed that breast cancer survivors have a wide range of Internet-based peer support options. The number of online communities identified in this review is an underestimation of the total number that exists for breast cancer survivors, given that we analyzed websites as whole entities. Many websites have multiple communities. In a separate study, we identified over 600 breast cancer groups on Facebook alone [20]. Likewise, the review conducted in 2003 that identified 546 online cancer 
Fig. 2 Launch dates of breast cancer-specific online communities $(N=33)$

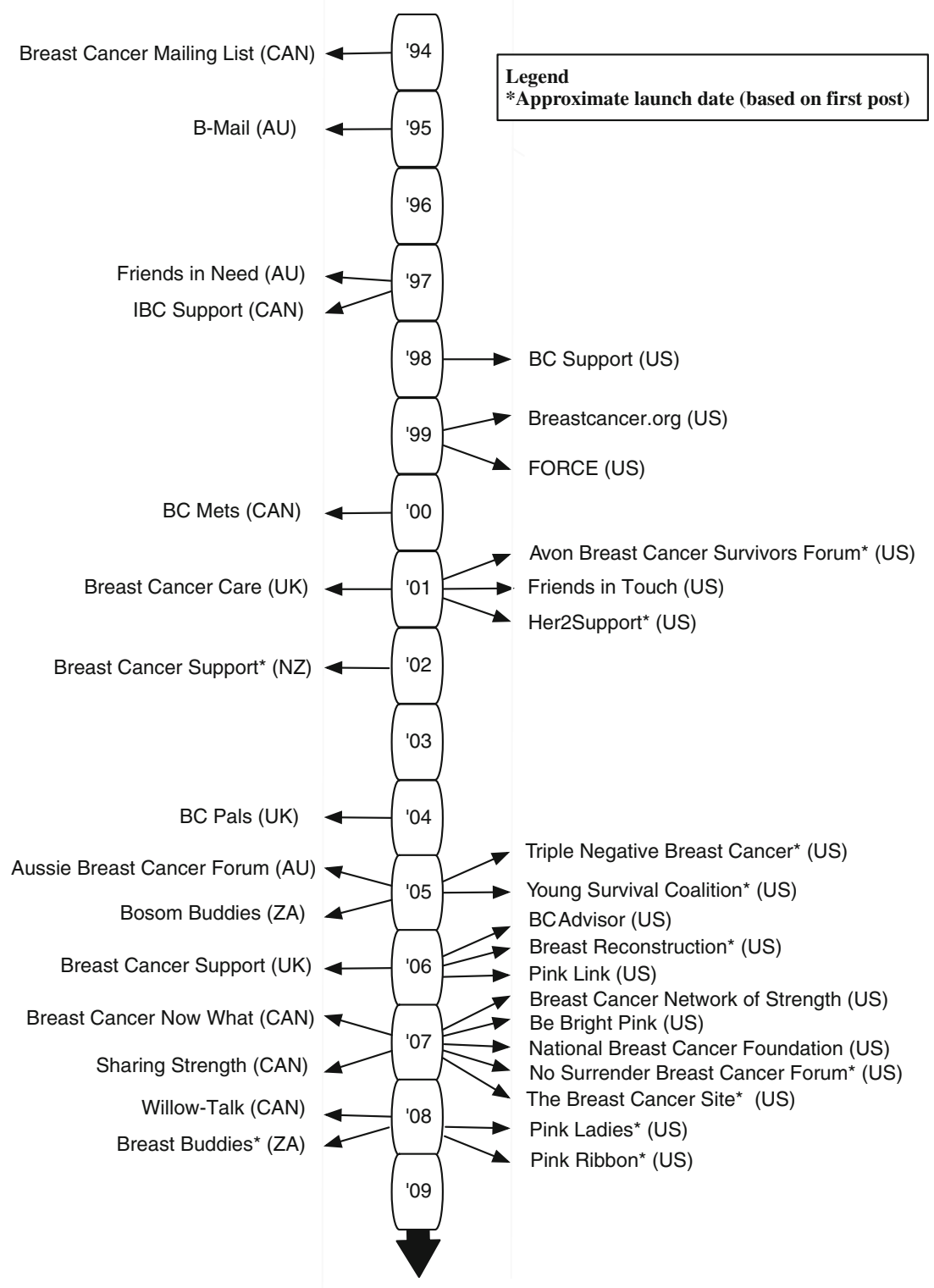

communities reported all of the 150 mailing lists offered by the Association of Online Cancer Resources at that time [14], a website included in our review. That 2003 review, although important, did not report the names or URLS of all the websites included in their sample, which limits further comparisons.
Our study found that the participation levels in online breast cancer communities varied from very high (e.g., more than one million posts) to very low (less than 15 posts). Ten sites accounted for $93.4 \%$ of the total number of message board posts in the breast cancer-specific websites sampled, each of which reported more than 100,000 posts. Interestingly,

Table 3 Activity level of breast cancer-specific online communities

\begin{tabular}{lllllll}
\hline & Number (websites) & Total & Min & Max & Median & IQR \\
\hline Total members & 22 & 140,162 & 10 & 78,931 & $1,118.5$ \\
Total posts & 30 & $4,186,275$ & 14 & $1,502,508$ & $19,669.5$ & $144,827.7$ \\
\hline
\end{tabular}

Data on membership size were available from 22 of the 38 breast cancer online communities, and data on site usage were available from 30 of the 38 breast cancer-specific online communities. All activity data were extracted on October 26, 2010

$I Q R$ interquartile range 
Fig. 3 Activity level of breast cancer-specific online communities

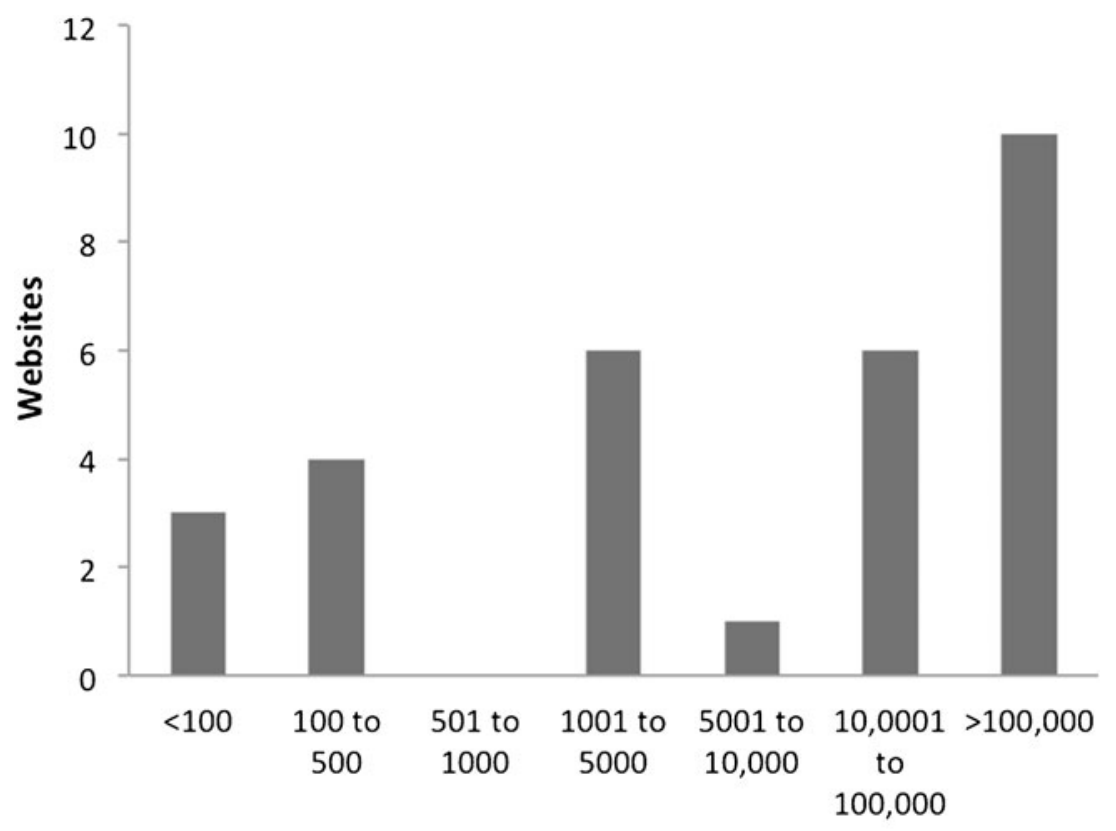

Message Board Posts four of the top ten sites catered to a less common type of breast cancer (e.g., hereditary) or characteristic of breast cancer survivor (e.g., young), demonstrating the potential of the Internet to leverage the "long-tail" [21]. The long-tail in health represents the numerous individuals with less common conditions that are poorly served by traditional support systems because they do not fit the model of the "average" patient [22]. Online communities enable these individuals to more easily connect, develop a critical mass, and build a shared knowledge base related to their condition [5].

Inadequate usage statistics prevented us from examining why these top ten sites were more popular than others. However, it is worthwhile to note that all top ten sites were initiated by a nonprofit organization, breast cancer survivor, or affected family caregiver as opposed to a commercial company, university, or health care institution. In fact, the majority of sites sampled that were exclusive to breast cancer were initiated by a breast cancer survivor or affected loved one (68\%), confirming anecdotal reports that have suggested that patients, and their loved ones, are taking a major role in creating health resources on the Internet [1, 23, 24]. According to a qualitative study with 23 webmasters of patient-initiated breast, fibromyalgia, and arthritis online communities, patients create such resources because of a desire to help others, promote awareness of the disease, or because existing resources do not meet their needs [25]. The personal motivation of the individual behind the online community may be a key success factor.

In our study, $7 \%$ of the online communities sampled were discontinued. Reasons cited for closure include changes in the strategic focus of the organization, inactivity, and spam (unsolicited messages that contain advertisements). Building successful, sustainable, and scalable online communities is
Table 4 Top ten most active breast cancer-specific online communities

\begin{tabular}{llc}
\hline Rank & Online community (URL) & Total posts $^{\mathrm{a}}$ \\
\hline 1 & Breastcancer.org (www.breastcancer.org) & $1,502,508$ \\
2 & Young Survival Coalition (www.youngsurvival.org) & 522,804 \\
3 & Breast Cancer Care UK (www.breastcancercare.org.uk) & 440,860 \\
4 & Susan G. Komen Foundation & 317,620 \\
5 & Breast Cancer Mailing List (www.bclist.org) & 308,160 \\
6 & FORCE-Facing Our Risk of Cancer Empowered (www.facingourrisk.org) & 276,091 \\
7 & Her2Support (www.her2support.org) & 185,302 \\
8 & BC Mets Mailing List (www.bcmets.org) & 132,609 \\
9 & Aussie Breast Cancer Forum (www.bcaus.org.au) & 119,679 \\
10 & BC Pals (www.bcpals.org/uk) & 105,594 \\
\hline
\end{tabular}


challenging, as we know little about the factors that determine their success [26]. Important sociotechnical attributes that warrant attention include anonymity, homophily (similarity of user experiences), presence (computer-simulated physical presence of others), interaction management (ability to control how one participates), and the sociability (ability to support social interactions) of the site [17, 24]. In addition, our study suggests that the success of online communities may depend on structural and sociopolitical factors such as the priorities of the organization, allocation of appropriate resources, and effective moderation.

Contrary to reports describing online communities as "ad hoc, self-organizing, organic discussions with no particular oversight or administration" [24], the majority of online communities in our sample were described as moderated or contained evidence of moderation by site administrators or volunteer community members. However, the extent and quality of the moderation appeared to vary with some sites suffering from a considerable amount of spam. Promoting the group, facilitating participation, responding to messages and removing spam are key to ensuring the success of an online community but are onerous activities consuming on average $10-15 \mathrm{~h}$ per week $[25,27]$. We need a better understanding on the role of moderation on the success and effectiveness of online communities and the resources required to support moderators in their roles.

Health professionals and patients have expressed concern about the potential for deception and misinformation on the Internet $[28,29]$, particularly in consumer-led online communities [30]. This study may serve as a further cause for concern, given that only one community sampled said they screened for accuracy before posting, and only a handful of sites engaged health-care professionals as moderators. However, there is evidence to suggest that online communities can effectively selfregulate content [31]. Content analysis of the un-moderated Breast Cancer Mailing List (which was included in our study) revealed that of 4,600 postings, only ten were found to be misleading or false, and seven of those were corrected by the community within $5 \mathrm{~h}$ of the original posting [32].

Overall, our study highlights a need for more welldesigned studies that investigate the factors associated with the success and effectiveness of unstructured, consumer-led online communities. Most of the effectiveness research to date has focused on online communities that are professionally led [7]. The only known RCT of an unstructured, un-moderated mailing list produced conflicting results and is limited by small sample and effect sizes. Participants, who were newly diagnosed breast cancer survivors, experienced a nonstatistically significant increase in distress over time. Paradoxically, $60 \%$ of participants reported high levels of satisfaction with the intervention, a subgroup of whom created their own mailing list at the end of the study [33]. These findings indicate a need for more exploratory research designs, as suggested by
Greenhalgh and Russell [34], which take into account the complex array of personal, social, political, and ideological factors that determine the success of eHealth interventions.

This inventory of online communities could serve as a guide for health-care professionals and a resource for breast cancer survivors. Surveys of breast and testicular cancer patients indicate that at least 20-35\% do not know of any online communities [35, 36], and a survey of oncologists and rheumatologists revealed that most found it challenging to provide up-to-date Internet sites to patients [37]. In the time elapsed since our last search, more resources for breast cancer survivors have emerged. Some of the most prominent are www.patientslikeme.org, www.ihadcancer.com, www.cancer connection.ca, and www.planetcancer.org. As no printed article would be able to provide an up-to-date inventory of sites, we have created an article on Wikipedia to complement this report. We invite interested researchers, developers, and survivors to edit it on an ongoing basis, thus co-creating the most accurate collection of breast cancer online communities.

\section{Limitations}

The search results are dependent on the terms included in the strategy and on the search engine used. We attempted to overcome this limitation by choosing common terms, two complementary strategies and the search engine with the greatest market share. A significant proportion of the online communities included in this review did not provide information on the date the site was launched or the usage statistics. Moreover, the launch dates reported in this paper should be regarded as estimates, given that it was not clear in all cases whether the communication feature (e.g., message board) was included on the site when it was first launched or if it was an add-on feature.

\section{Conclusions}

There is a wide range of online communities available for breast cancer survivors with extensive archives of personal illness experiences. The scope of online peer support options will inevitably increase due to the growing popularity of social media applications that provide the public with the tools to create the support resources that they need. Future efforts should focus on identifying the factors that determine their success and effectiveness.

Acknowledgments This work is a component of JLB's PhD thesis at the University of Toronto. JLB has full control over all primary data and agrees to allow the journal to review the data if requested. JLB wishes to acknowledge the guidance of her PhD thesis committee: LE Ferris PhD CPsych LLM (ADR) LLM (Admin Law), J Katz PhD CPsych, and AR Jadad MD DPhil FRCPC. This work was supported by CIHR Strategic Doctoral Fellowships in Health Care, Technology and Place, and Cell Signaling in Mucosal Inflammation and Pain. 
Conflict of interest None.

\section{References}

1. Fox S (2011) Social life of health information: Pew Internet and American Life Project, 5/2011. http://pewinternet.org/Reports/ 2011/Social-Life-of-Health-Info.aspx

2. Ziebland S, Wyke S (2012) Health and illness in a connected world: how might sharing experiences on the internet affect people's health? Milbank Q 90(2):219-49

3. Statistics Canada (2010) Canada internet use survey: the daily. http://www.statcan.gc.ca/daily-quotidien/110525/dq110525beng.htm. Accessed April 6, 2012

4. Preece J (2000) Online communities: designing usability, supporting sociability. Wiley, New Work

5. Bender JL, O'Grady L, Jadad AR (2008) Supporting cancer patients through the continuum of care: a view from the age of social networks and computer-mediated communication. Curr Oncol s107:s42-7

6. Davison KP, Pennebaker JW, Dickerson SS (2000) Who talks? The social psychology of illness support groups. Am Psychol 55 (2):205-17

7. Eysenbach G, Powell J, Englesakis M, Rizo C, Stern A (2004) Health related virtual communities and electronic support groups: systematic review of the effects of online peer to peer interactions. BMJ 328(7449): 1166

8. Hoybye MT, Johansen C, Tjornhoj-Thomsen T (2005) Online interaction. Effects of storytelling in an internet breast cancer support group. Psycho-Oncology 14(3):211-20

9. Sharf BF (1997) Communicating breast cancer on-line: support and empowerment on the Internet. Women Health 26(1):65-84

10. Shaw B, McTavish F, Hawkins R et al (2009) Experiences of women with breast cancer: exchanging support over the CHESS network. J Health Commun 5:135-159

11. Vilhauer RP (2009) Perceived benefits of online support groups for women with metastatic breast cancer. Women \& Health 49(5):381404

12. van Uden-Kraan CF, Drossaert CH, Taal E et al (2008) Empowering processes and outcomes of participation in online support groups for patients with breast cancer, arthritis, or fibromyalgia. Qual Health Res 18(3):405-17

13. Rogers S, Chen Q (2005) Internet community group participation: psychosocial benefits for women with breast cancer. J Comp Med Commun 10(4):1

14. Im EO, Chee W, Tsai HM et al (2005) Internet cancer support groups: a feminist analysis. Cancer Nurs 28(1):1-7

15. Hoffman-Goetz L, Clarke JN (2000) Quality of breast cancer sites on the World Wide Web. Cdn J Public Health 91(4):281-4

16. Meric F, Bernstam EV, Mirza NQ et al (2002) Breast cancer on the world wide web: cross sectional survey of quality of information and popularity of websites. BMJ 324(7337):577-81

17. Preece J (2001) Sociability and usability in online communities: determining and measuring success. Behav Inform Tech 20(5):347-356

18. NetMarketShare.com (2009) Search engine market share for December 2009: http://www.netmarketshare.com/search-engine-marketshare. aspx?qprid=4. Accessed December 15, 2009
19. Ussery B (2008) Google: average number of words per query have increased: Beu Blog 2008 http://www.beussery.com/blog/ index.php/2008/02/google-average-number-of-words-per-queryhave-increased/ Accessed December 15, 2009

20. Bender J, Jimenez-Marroquin MC, Jadad AR (2011) Seeking support on Facebook: a content analysis of breast cancer groups. J Med Internet Res 13(1):e16

21. Anderson C, The long tail. Wired 2004; October 12:10: http:// www.wired.com/wired/archive/12.10/tail.html. Accessed April 15,2012

22. Deshpande A, Jadad AR (2006) Web 2.0: could it help move the health system into the 21st century. J Men's Health Gend 3(4):332-336

23. Bass SB (2003) How will Internet use affect the patient? A review of computer network and closed Internet-based system studies and the implications in understanding how the use of the Internet affects patient populations. J Health Psychol 8(1):25-38

24. Walther JB, Pingree S, Hawkins RP (2005) Attributes of interactive online health information systems. J Med Internet Res 7(3):e33

25. van Uden-Kraan CF, Drossaert CHC, Taal E (2010) Patientinitiated online support groups: motives for initiation, extent of success and success factors. J Telemed Telecare 16(1):30-34

26. Kairam S, Wang DJ, Leskovec J (2012) The life and death of online groups: predicting group growth and longevity. Presented at WSDM, Seattle, Washington, USA Febrary 8-12, 2012

27. O'Grady LA, Bender JL, Wiljer D et al (2010) Promoting and participating in online health forums: a guide to facilitation and evaluation for health professionals. J Commun Healthcare 3(4):246-257

28. Jadad AR, Gagliardi A (1998) Rating health information on the Internet: navigating to knowledge or to Babel? JAMA 279(8):611-4

29. Gagliardi A, Jadad AR (2002) Examination of instruments used to rate quality of health information on the Internet: chronicle of a voyage with unclear destination. BMJ 324(7337):569-573

30. Broom A (2005) Virtually he@lthy: The impact of internet use on disease experience and the doctor-patient relationship. Qual Health Res 15(3):325-45

31. Jadad AR, Enkin MW, Glouberman S (2006) Are virtual communities good for our health? BMJ 332(7547):925-926

32. Esquivel AF, Meric-Bernstam F, Bernstam EV (2006) Accuracy and self correction of information received from an internet breast cancer list: content analysis. BMJ 332(7547):939-42

33. Salzer MS, Palmer SC, Kaplan K et al (2010) A randomized controlled study of internet peer-to-peer interactions among women newly diagnosed with breast cancer. Psychooncol 19(4):441-6

34. Greenhalgh T, Russell R (2010) Why do evaluations of eHealth programs fail? An alternative set of guiding principles. PLoS Med 7(11):e1000360

35. Bender JL, Wiljer D, To MJ et al (2012) Testicular cancer survivors' supportive care needs and use of online support: a cross-sectional survey. J Suppor Care Cancer 20(11):2737-46. doi:10.1007/s00520012-1395-x

36. Bender JL (2011) The web of support: a multi-method study examining the role of online communities as a source of peer-topeer supportive care for breast cancer survivors: Doctoral Dissertation, University of Toronto

37. van Uden-Kraan CF, Drossaert CHC, Taal E et al (2010) Experiences and attitudes of Dutch rheumatologists and oncologists with regard to their patients' health related Internet use. Clin Rheumatol 29(11):1229-36 